Letters to the Editors should be addressed to the Editor, APPLIED OPTICS, AFCRL, Bedford, Mass. 01730. If authors will state in their covering communications whether they expect their institutions to pay the publication charge, publication time should be shortened (for those who do).

\section{Proposed Distributed Feedback Crystal Cavities for X-Ray Lasers}

\section{Elachi, G. Evans, and F. Grunthaner}

California Institute of Technology, Jet Propulsion Laboratory, Pasadena, California 91103.

Received 28 May 1974.

The strong interest in the coherent generation and guiding of $\mathrm{x}$ rays is well known. Many papers have recently appeared treating different concepts of stimulated $\mathrm{x}$-ray emissions, ${ }^{1-3}$ and $x$-ray guiding in thin films was achieved. ${ }^{4}$ Crystals were suggested as end reflectors to generate feedback. ${ }^{1}$ Here we suggest a different type of cavity using zeolite crystals that would guide the emitted $x$-rays and at the same time generate the necessary feedback for self-sustained oscillation.

Zeolite crystals ${ }^{5-7}$ are aluminosilicate porous structures, commonly termed molecular sieves, with one-, two-, or three-dimensional channels having minimum pore diameters of 3-12 $\AA$. These channels are formed by the juncture of larger sodalite cage units that are arranged with a peri-

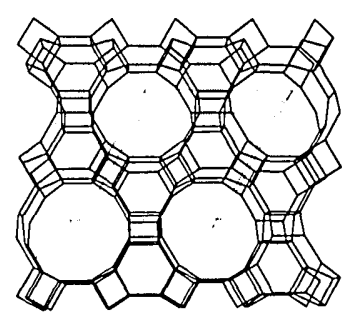

(a)

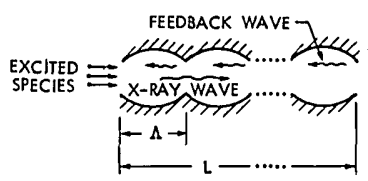

(C)

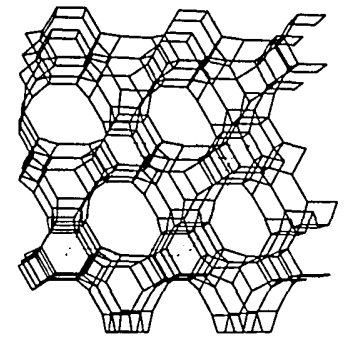

(b)

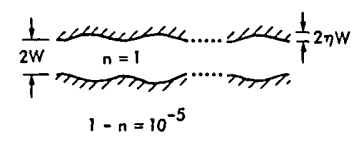

(d)
Fig. 1. (a) Skeletal framework of Gmelinite viewed along [001]. (b) Skeletal framework of Offretite viewed along [001]. (c) Sketch of a cut along one of the channels. The cross section changes with a period $\Lambda$. (d) Theoretical analysis can be applied to a simplified case where the boundary perturbation is sinusoidal. The perturbation $2 \eta W$ is equal to the first coefficient of the Fourier expansion of the boundary geometry shown in (c). odicity analogous to that of simple atomic and molecular crystals. The internal diameter of the composite sodalite cage can range from $6 \AA$ to $24 \AA$ as a function of the mineral. Considerable porosity to gases is exhibited by these structures. Thus excited gases or plasmas can be forced inside these pores by a pressure differential. In Fig. 1 we sketched some zeolite crystal configurations.

Because of the higher index of refraction in the channel ( $n=1$ for vacuum, and $1-n \sim 4 \times 10^{-5}$ for aluminosilicates in the soft $\mathrm{x}$-ray region) the $\mathrm{x}$-ray waves could be guided in the channels. X-ray guiding was recently demonstrated ${ }^{4}$ in thin films. The periodic change in the channel cross section would generate Bragg-type reflection of the wave, which leads to distributed feedback (DFB). DFB cavities are already used in optical lasers. ${ }^{8,9}$ There are three oscillation requirements. (1) The period of the structure is an integer times half the $\mathrm{x}$-ray wavelength (Bragg condition). This can be easily satisfied in the soft $\mathrm{x}$-ray region due to the availability of a large number of zeolite crystals with different periodicities. Some typical crystal parameters are given in Table I. (2) The gain is larger than the losses $\sigma$ in the aluminosilicate material, which is of the order of $\sigma \sim 10-100 \mathrm{~cm}^{-1}$. Bohn ${ }^{2}$ computed the gain for soft x-ray transitions in hydrogenlike ions, and he reported gain coefficient values well above $100 \mathrm{~cm}^{-1}$. (3) The feedback is strong enough to generate self-oscillation. This condition is hard to verify analytically, but it seems that the drastic change in the channel diameter (Table I) would provide a strong feedback.

Thus we conclude that, althought it is not an easy task, zeolite crystals should be seriously considered as candidates for soft $\mathrm{x}$-ray cavities.

This paper represents the results of one phase of research carried out at the Jet Propulsion Laboratory, California Institute of Technology, under contract NAS7-100 sponsored by the National Aeronautics and Space Administration.
Table I. Parameters of Some Typical Zeolite Crystals

\begin{tabular}{lccc}
\hline Zeolite & $\begin{array}{c}\text { Minimum } \\
\text { channel } \\
\text { diameter }(\AA)\end{array}$ & $\begin{array}{c}\text { Cage } \\
\text { diameter } \\
(\AA)\end{array}$ & $\begin{array}{c}\text { Channel } \\
\text { contractness } \\
\text { periodicity }(\AA)\end{array}$ \\
\hline Erionite & $3.6-5.2$ & 15 & $13-15$ \\
Mordinite & $5.7-7.0$ & $13-18$ & $7.29-7.52$ \\
Ferrierite & $3.4-5.5$ & $12-15$ & $7.3-7.5$ \\
Faujasite & $7.3-7.5$ & $15-18$ & 24.7 \\
\hline
\end{tabular}




\section{References}

1. A. G. Molchanov, Sov. Phys.-Usp. 15, 124 (1972).

2. W. L. Bohn, Appl. Phys. Lett. 24, 16 (1974).

3. I. Freund, Appl. Phys. Lett. 24, 13 (1974).

4. E. Spiller and A. Segmuller, Appl. Phys. Lett. 24, 60 (1974).

5. J. V. Smith, Mineral Soc. Am. Spec. Paper 1, 281 (1963).

6. K. Fischer and W. M. Meier, Fortschr. Mineral 42, 50 (1965).

7. W. M. Meier, S.C.I. Monograph Mol. Sieves 10 (1968).

8. H. Kogelnik and C. V. Shank, J. Appl. Phys. 43, 2327 (1973).

9. D. P. Schinke, R. G. Smith, E. G. Spender, and M. F. Gavin, Appl. Phys. Lett. 21, 494 (1972).

\section{Methods for Compensating the Nonlinearity in the Diffraction Angle in $\mathrm{TeO}_{2}$ Abnormal Bragg Deflectors}

\section{Kawabuchi, A. Fukumoto, and H. Hayami}

Matsushita Research Institute Tokyo, Inc., Ikuta, Kawasaki, Kanagawa, Japan.

Received 3 September 1974.

A $\mathrm{TeO}_{2}$ abnormal Bragg deflector (ABD) ${ }^{1}$ excells other acoustooptic light deflectors in its diffraction efficiency and the number of resolvable spots, although it has a simple structure. Unfortunately, the $\mathrm{TeO}_{2} \mathrm{ABD}$ had a strong nonlinearity in the diffraction angle against the acoustic frequency, which is undesirable for applications.

In the case of a random access mode of operation, the $\mathrm{TeO}_{2} \mathrm{ABD}$ causes tracking errors in the deflection position, if the $\mathrm{TeO}_{2} \mathrm{ABD}$ is operated by applying discrete acoustic frequencies with equal separations for obtaining the deflection position with equal separation. The method to correct such tracking errors is not difficult, i.e., the discrete frequency generators are so adjusted that they compensate the nonlinearity in the diffraction angle.

In a linear FM mode of operation, in which the acoustic frequency is swept continuously, the nonlinearity in the diffraction angle causes distortions in the light spots scanned. The calculated relation between the acoustic frequency $f$ and the frequency derivative of the diffraction angle $\theta_{d},\left(d \theta_{d} / d f\right)$, normalized by that at the Bragg center frequency $f_{0}$, is shown in Fig. 1 for three optical wavelengths.

There is another point that should be considered in using a $\mathrm{TeO}_{2} \mathrm{ABD}$ operated in a linear FM mode. This is the cy-

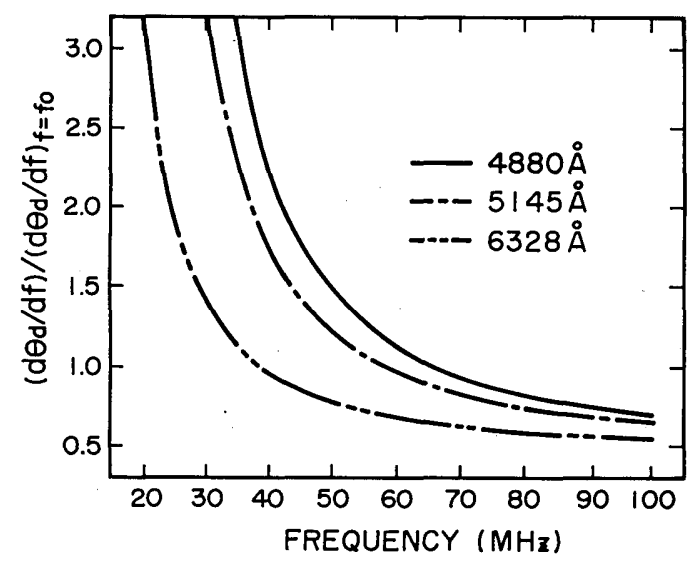

Fig. 1. Frequency variation of the light deflection speed on screen vs the acoustic frequency.

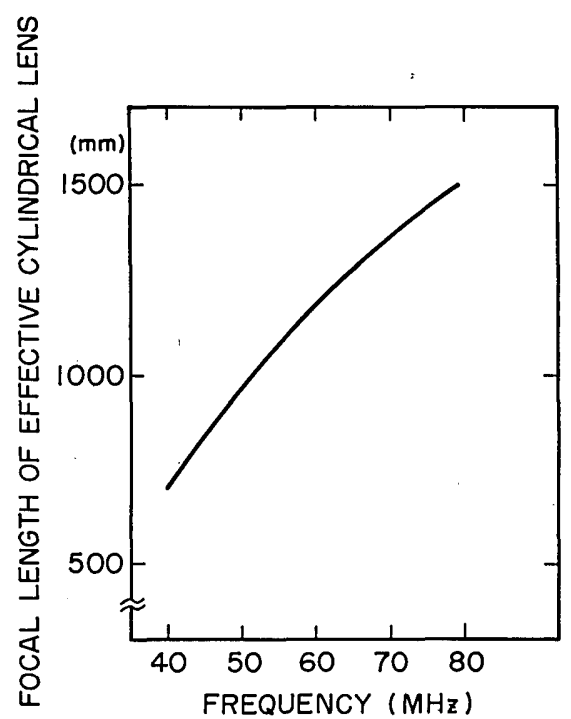

Fig. 2. Variation of the focal length of the effective cylindrical lens in abnormal Bragg diffraction in $\mathrm{TeO}_{2}$.

lindrical lensing effect in the acoustooptic light deflector. As the velocity $v$ of the sound propagating along the [110] axis of a $\mathrm{TeO}_{2}$ crystal is very slow, $0.62 \times 10^{5} \mathrm{~cm} / \mathrm{sec}$, the cylindrical lensing effect is remarkable. The cylindrical lensing effect and the method for compensation were studied by Dickson ${ }^{2}$ for normal Bragg deflectors. As mentioned before, the $\mathrm{TeO}_{2} \mathrm{ABD}$ has a strong nonlinearity in the diffraction angle, accordingly the effective focal length $f_{c}$ of the ABD is given by Eq. (1):

$$
f_{c}=v /\left[\left(d \theta_{d} / d f\right)(d f / d t)\right] .
$$

In Eq. (1), $t$ is the time. The calculated relation between the effective focal length and the acoustic frequency in a $\mathrm{TeO}_{2} \mathrm{ABD}$ is shown in Fig. 2, in which the sweep range is from $40 \mathrm{MHz}$ to $79 \mathrm{MHz}$, sweep time $58.5 \mu$ sec, optical aperture $4 \mathrm{~mm}$, and optical wavelength $5145 \AA$. In Fig. 2, the value in the abscissa represents the lowest acoustic frequency in the optical aperture of the deflector. Figure 2 shows that the effective focal length varies so remarkably that the method of compensation proposed by Dickson, i.e., the use of a cylindrical lens having a fixed focal length, is not sufficient for $\mathrm{TeO}_{2} \mathrm{ABD}$.

This variation in the effective focal length should also be compensated. This can be performed by generating a properly shaped sawtooth wave $(d f / d t)$ to the FM sweeper, which compensates the nonlinearity in the $\left(d \theta_{d} / d f\right)$ in Eq. (1) making [ $\left.\left(d \theta_{d} / d f\right)(d f / d t)\right]$ a constant. After the compensation by this method, the cylindrical lensing effect must be corrected by Dickson's method.

Consequently, the distortions in the light spots scanned and the cylindrical lensing effect are all corrected. The methods proposed in this paper were appreciated experimentally using $\mathrm{TeO}_{2} \mathrm{ABD}$.

The authors are grateful to T. Ose and I. Ogura, Institute of Industrial Science, University of Tokyo, for discussions and suggestions.

\section{References}

1. A. W. Warner et al., J. Appl. Phys. 43, 4489 (1972).

2. L. D. Dickson, Appl. Opt. 11, 2196 (1972). 